\title{
RESPUESTA CARDIOVASCULAR EN EL TRAUMA RAQUIMEDULAR
}

\author{
EduARdo LeMA FloREZ, M.D. ${ }^{1}$, LUIS ALBERTO TAFUR B, M.D. ${ }^{2}$
}

\section{RESUMEN}

La respuesta cardiovascular en el trauma raquimedular (TRM) se debe fundamentalmente a la respuesta adrenérgica del sistema nervioso autónomo, lo que se puede manifestar como bradi-arritmias, vasodilatación y colapso cardiovascular, entre otros. La comprensión de la fisiopatología de la respuesta cardiovascular permitirá entender las medidas preventivas y/o terapéuticas que debe establecer para el manejo de los estados que amenazan la estabilidad y la vida del paciente. Este conocimiento debe ser de manejo global entre el personal de salud, dado que, aun cuando la epidemiología del TRM varía de una país a otro, principalmente en las causas, la frecuencia del mismo hace muy probable que cualquier profesional de salud pueda verse enfrentado a atender a un paciente con TRM en la fase aguda o crónica aun que es claro que los pacientes con TRM con riesgo alto de complicaciones cardio pulmonares pueden mejoran su resultado final al ser manejados, en la fase inicial, en unidades de cuidados intensivos con capacidad de soporte cardio pulmonar y ventilatorio.

\section{Palabras claves: Respuesta cardiovascular, Trauma raquimedular}

\section{INTRODUCCIÓN}

La respuesta cardiovascular en el trauma raquimedular (TRM) se debe fundamentalmente a la respuesta adrenérgica del sistema nervioso autónomo (SNA), lo que se puede manifestar como bradi-arritmias, vasodilatación y colapso cardiovascular, entre otros; La comprensión de la fisiopatología de la respuesta cardiovascular permitirá al anestesiólogo entender las medidas preventivas y/o terapéuticas que debe establecer para el manejo de los estados que amenazan la estabilidad y la vida del paciente.

${ }^{1}$ Médico Anestesiólogo. Profesor. Universidad del Valle, Hospital Universitario del Valle. SEGANEST, Médico Anestesiólogo Instituto Para Niños Ciegos y Sordos del Valle del Cauca. Cali, Colombia ${ }^{2}$ Profesor asistente. Coordinador de Neuroanestesia. Departamento de Anestesiología. Universidad del Valle. Cali, Colombia

\section{SUMMARY}

The cardiovascular response in spinal cord trauma (SCT) is mainly due to the adrenergic response of the autonomic nervous system, which can manifest as brady-arrhythmias, vasodilation and cardiovascular collapse, among others. The understanding of the pathophysiology of cardiovascular response enable you to understand the preventive and / or therapeutic measures should be established to manage the states that threaten the stability and life of the patient. This knowledge must be global management among health personnel, since even if the epidemiology of SCT varies from one country to another, mainly in the causes, frequency of it makes it very likely that any health professional may be facing caring for a patient with SCT in acute and chronic phase or even that it is clear that patients with SCT at high risk of cardiovascular complications lung can improve end result to be handled in the initial phase, in intensive care units with capacity cardio pulmonary and ventilatory support.

\section{Key words: Cardiovascular response, Spinal cord trauma}

Este conocimiento debe ser de manejo global entre el personal de salud, dado que, aun cuando la epidemiología del TRM varía de una país a otro, principalmente en las causas, la frecuencia del mismo hace muy probable que cualquier profesional de salud pueda verse enfrentado a atender a un paciente con trauma raquimedular en la fase aguda o crónica aun que es claro que los pacientes con TRM con riesgo alto de complicaciones cardio pulmonares pueden mejoran su resultado final al ser manejados, en la fase inicial, en unidades de cuidados intensivos con capacidad de soporte cardio pulmonar y ventilatorio.

\section{OBJETIVO}

El objetivo de este escrito es recordar al anestesiólogo las bases fisiológicas pertinentes para la comprensión y el manejo de las manifestaciones cardiovasculares que pueden amenazar la vida del paciente con TRM. 


\section{SNA, ENFOQUE DESDE LAFUNCIÓN MEDULAR}

No sobra recordar que los cuerpos de las fibras pre ganglionares del SNS se encuentran en la columna intermedio lateral de la medula espinal y que esto ocurre en los segmentos espinales desde T1 hasta L1 o L2, por lo cual el SNS es considerado toraco-lumbar, mientras que el SNP la fibra pre ganglionar tiene su origen en el tallo cerebral emergiendo del cráneo por los pares craneales III, VII, IX y X, siendo el X el más importante involucrado en la respuesta cardiovascular en el TRM.
Otra porción del parasimpático tiene su origen en la columna intermedio lateral de los segmentos S2 a S4 que participa en la regulación de la función urogenital.Figura 1. Este "recordar" resulta pertinente por cuanto ayuda a entender tanto los efectos como los signos clínicos cardio vasculares de la lesión medular, lo cual puede mejorar el seguimiento de la efectividad de las medidas terapéuticas y el manejo clínico.

De una manera sencilla, podemos resumir que la inervación simpática de los niveles espinales T1 a T5

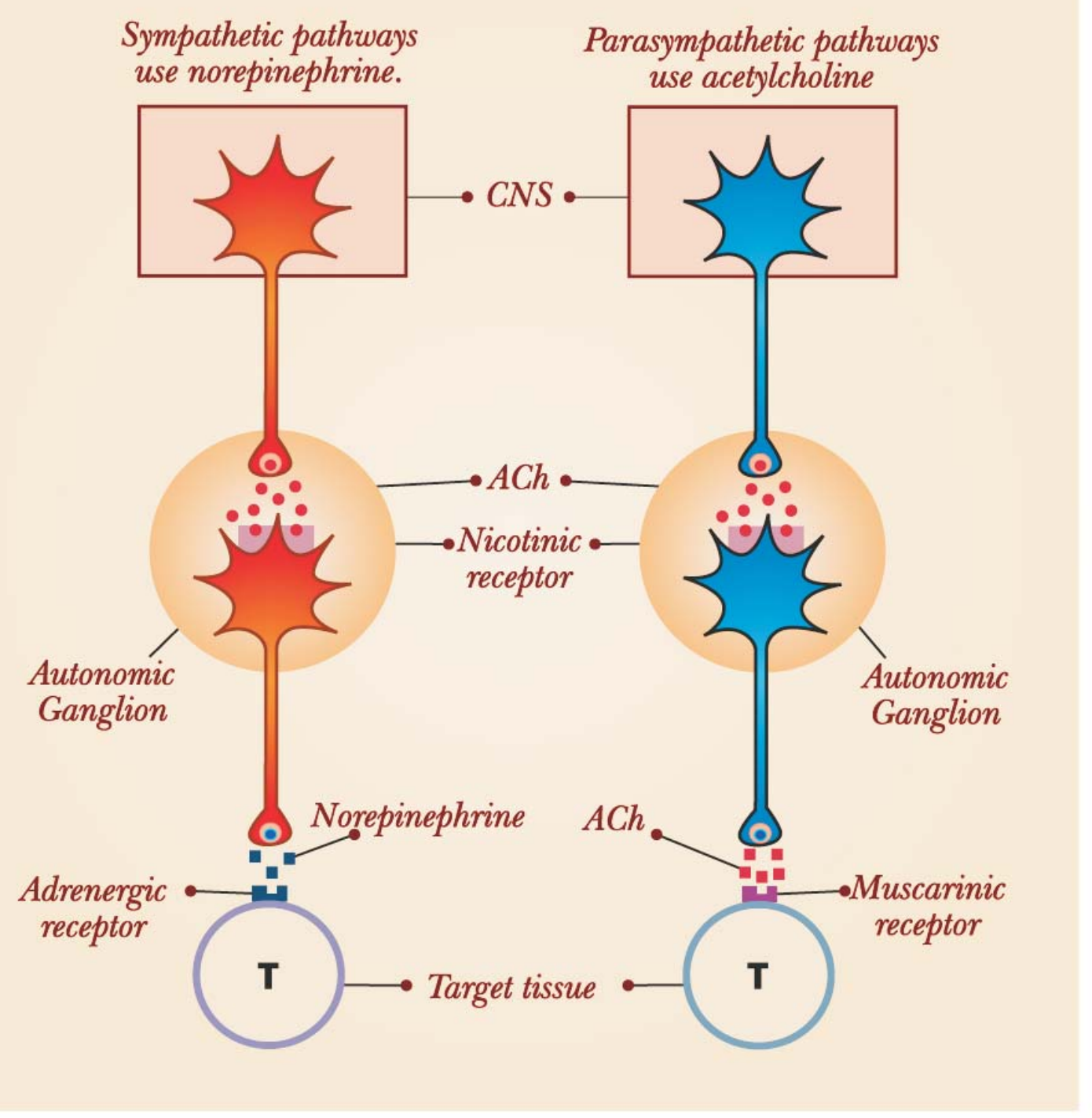

Figura 1. Esquema del sistema nervioso autónomo (simpático y parasimpático) 
regula el tono vascular de la parte superior del cuerpo y estimula el nodo sino atrial, mientras que los segmentos T5 a L2, el tono vascular de la parte inferior del cuerpo, especialmente, del lecho esplácnico donde se encuentra aproximadamente el $25 \%$ de la volemia.

El Parasimpático, a través del nervio vago, regula hacia abajo la frecuencia del Nodo sino auricular, la aurícula y fibras de Purkinje (disminuyendo no solo la frecuencia cardíaca, sino también la contractilidad) y el tono vascular de algunos órganos como glándulas salivales, gastrointestinales y cuerpo cavernoso.Figura 2.

Establecer cuál es la función autonómica residual de un paciente después de un TRM ayuda al anestesiólogo a definir las medidas terapéuticas y la valoración de su efectividad. Una forma práctica puede ser usar el score publicado por la "International SpinalCordSociety", el cual se basa en la evaluación y seguimiento del control autonómico del corazón, la presión arterial, la sudoración, termorregulación, sistema broncopulmonar y del tracto urológico inferior, la vejiga y la función sexual.

\section{MANIFESTACIONES CARDIOVASCULARES EN EL TRM}

Se puede afirmar que los dos extremos del espectro de las manifestaciones cardiovasculares del TRM lo representan la disreflexia autonómica, la enfermedad coronaria y arterioesclerosis sistémica (fase crónica principalmente, aun cuando se describe desde los primeros días del trauma) y el shock medular (fase aguda).

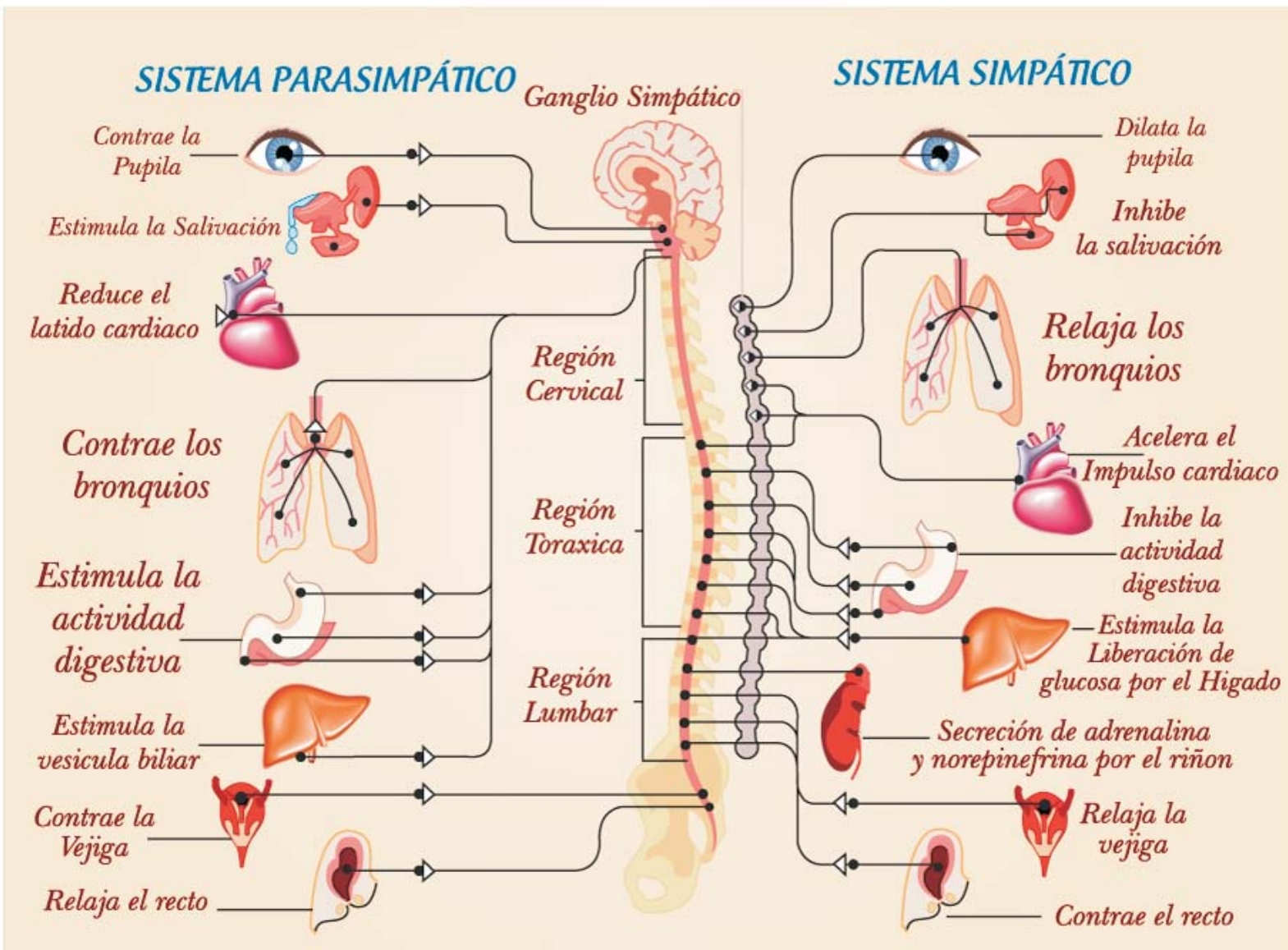

Figura 2. Representación de los órganos efectores y su relación con el sistema nervioso 


\section{CHOQUE ESPINAL}

Durante los primeros minutos del trauma, ocurre una descarga adrenérgica de la medula suprarrenal, con hipertensión y bradicardia o taquiarritmias; 3 o 4 minutos después empieza a disminuir todo y en aquellos pacientes con lesión medular alta aparece el shock espinal, el cual puede durar hasta 4 a 12 semanas. Aproximadamente entre el 7 y el $10 \%$ de todos los pacientes con un TRM desarrollan un shock neurogénico circulatorio en el cual debe pensarse en presencia de hipotensión y bradicardia. Es importante realizar el diagnóstico diferencial con el shock hipovolémico. Tabla 1.

Es decir que una lesión medular espinal podrá producir vasodilatación con disminución de la precarga por "secuestro" de la volemia en el lecho esplácnico, con bradicardia, hipotermia, disritmias y disminución de la contractilidad miocárdica por estímulo parasimpático sin oposición del SNS simpático, según el nivel de la lesión. En el paciente poli traumatizado, reconocer este cuadro resulta de fundamental importancia dentro de las opciones diagnósticas para evitar operar a un paciente inestable por la lesión medular o dejar abandonada una lesión quirúrgica explicando la situación de inestabilidad por el TRM. Para esto resulta útil la búsqueda del reflejo bulbo cavernoso, el cual es mediado por las raíces sacras ya descritas. La prueba consiste en observar la contracción del esfínter anal tras realizar presión sobre el glande o la vulva. Su ausencia en el paciente inestable indica la presencia de shock medular y su reaparición, es el primer signo de resolución del shock.
La hipotensión ortostática es más frecuente en pacientes con lesiones espinales altas y requiere de especial atención pues se presenta con los cambios de posición del paciente y puede resultar de muy difícil manejo. Los pacientes con lesiones más bajas presentan también caídas de presión arterial. En ambos casos estos cambios se presentan independientemente de si la lesión es completa o incompleta.

Para el manejo del shock, debe realizarse un buen diagnóstico y un plan de soporte hemodinámico. En general se recomienda que en los pacientes con TRM debe procurarse en la fase aguda una PAM $>85 \mathrm{mmHg}$, aun con la ayuda de vas opresores, por al menos una semana lo cual puede mejorar el resultado final del paciente,,Dado el impacto de estas complicaciones en el resultado final de estos pacientes, resulta razonable recomendar que aquellos pacientes con riesgo cardio pulmonar sean monitorizados en la fase aguda en una unidad de cuidados intensivos. La bradicardia extrema puede manejarse con atropina. Esta medida, en presencia de una adecuada carga de líquidos endovenosos puede resultar efectiva en el manejo de la hipotensión. La efedrina resulta útil para el manejo de la hipotensión en dosis de $5 \mathrm{mg}$ intravenosos o 25 a $50 \mathrm{mg}$ subcutáneos cada 4 a 6 horas.

\section{DISREFLEXIAAUTONÓMICA}

La Disreflexia autonómica es un cuadro que puede presentarse aún desde las etapas más tempranas del TRM hasta en el estado crónico con una lesión medular incompleta o completa y puede convertirse en un evento muy grave y aún mortal. Tras un estímulo por

Tabla 1

Diagnóstico diferencial del shock espinal y shock hipovolémico

\begin{tabular}{|c|c|c|}
\hline & SHOCK HIPOVOLEMICO & SHOCK NEUROGENICO \\
\hline PRESION ARERIAL & BAJA & BAJA \\
\hline FRECUENCIA CARDIACA & TAQUICARDIA & BRADICARDIA \\
\hline TEMPERATURA DE LA PIEL & FRIO & TIBIO \\
\hline REFLEJOS ESPINALES & $\begin{array}{l}\text { PRESENTES (EXCEPTO } \\
\text { CUANDO COEXISTEN) }\end{array}$ & AUSENTES \\
\hline
\end{tabular}


debajo del nivel de la lesión medular (Vejiga distendida en el $85 \%$ de los casos, imputación fecal en el 13 a 19\%, apendicitis, estímulo quirúrgico, actividad sexual, entre otras), ocurre una descarga de catecolaminas que produce vasoconstricción y aumento de la presión arterial, el cuál es sensado por los baro receptores generando una respuesta moduladora de origen central únicamente por encima del nivel de la lesión. El parto en pacientes de riesgo se asocia con un 85 a $90 \%$ de riesgo de desarrollar disautonomía. De esta forma, el estímulo adrenérgico persistirá por debajo del nivel de la lesión (vaso constricción, palidez, hipertensión) y la respuesta moduladora producirá flush, congestión, bradicardia, por encima del nivel de la lesión. Este evento aparece principalmente en lesiones medulares altas, por encima de T6, esto es, nivel sensitivo sexto espacio intercostal o apéndice xifoides, dado que la evaluación del nivel motor entre los segmentos T2 a L2 resulta muy difícil Un síntoma muy precoz lo representa la cefalea, de tal manera que cuando un paciente con una lesión medular alta presenta cefalea e hipertensión (aumento de un 20\% de la PAM basal) debe pensarse de inmediato en una disreflexia y realizar la búsqueda y manejo del evento desencadenante como inicio del tratamiento, seguido de vasodilatadores de acción rápida (nitroglicerina) cuando esto no es suficiente o cuando la presión sistólica está por encima de $150 \mathrm{mmHg}$. Cambiar la posición del paciente de manera transitoria hacia un Fowler, pude ayudar a disminuir la presión arterial de manera rápida. El principal riesgo del manejo es la hipotensión, por lo cual la presión arterial debe ser monitoreada de manera constante hasta resolver el evento. Otros signos de la disautonomía son el "flushing" facial, diaforesis por encima del nivel de la lesión, visión borrosa, ansiedad y congestión nasal.

En el paciente anestesiado, la sospecha de DA es fundamental para el diagnóstico temprano. Un incremento súbito de la presión arterial sistólica de 20 a $40 \mathrm{mmHg}$ puede indicar el inicio de una disreflexia autonómica. La aparición de taquicardia, seguida de bradicardia refleja con o sin alteraciones de la onda $\mathrm{T}$, arritmias, fibrilación ventricular pueden desencadenarse también. El paro cardíaco durante el intra operatorio en un paciente con lesión medular alta (por encima de T6) debe ser el diagnóstico a considerar con miras a establecer su manejo. Para el manejo inicial, resulta fundamental la identificación y cese del estímulo desencadenante. La medida preventiva y terapéutica más efectiva es lograr una adecuada profundidad anestésica durante la intervención aun cuando esta sea por debajo del nivel de lesión, evitar y diagnosticar distensión vesical e impactación fecal y estímulos nociceptivos por debajo de la lesión (compresión por ejemplo).

Tanto en la fase aguda, como en la crónica, el anestesiólogo debe estar atento a los eventos de hipotensión asociados a los cambios de posición. La hipotensión ortostática es de origen multi causal e incluye no solo el compromiso simpático, sino, el compromiso del sistema renina angio tensina, bajos niveles plasmáticos de catecolaminas, función alterada de los baro receptores, hiponatremia e hipovolemia, entre otros.

\section{TROMBOSIS VENOS PROFUNDA $Y$ TROMBO EMBOLISMO PULMONAR (TVP/TEP)}

Otro evento de origen vascular que representa una amenaza para la vida en este grupo de pacientes lo representa el riesgo aumentado de desarrollar TVP y TEP en los pacientes con TRM, debido principalmente, a la pérdida de la actividad de bomba que tiene el músculo esquelético de las extremidades inferiores (bombas impulso-aspirativas "BIAS") y al desacondicionamiento cardiovascular y a estados de hipercoagulabilidad, los pacientes con trauma raquimedular tienen mayor riesgo que la población general de presentar una trombosis venosa profunda y embolismo pulmonar.

La profilaxis tanto mecánica como farmacológica con heparinas de bajo peso molecular debe ser considerada durante los primeros tres meses del trauma como medida preventiva de TVP/TEP. La trombo profilaxis mecánica es mandatorio en virtualmente todos los pacientes con TRMy resulta útil además de su efecto mecánico, porque estimula la actividad fibrinolítica endógena.Esto justifica la intervención de un equipo profesional especializado en el manejo de estos pacientes.

Uno de los objetivos de la cirugía temprana para estabilización ósea de la columna vertebral, es la posibilidad de iniciar de manera temprana la terapia kinésica activa. Los hábitos alimenticios y el control de peso, son también medidas importantes en la prevención de la TVP/TEP en el paciente con TRM. 
Existe poca literatura en cuanto al manejo del trauma raquimedular en niños comparado con la disponible para los adultos. Los principios de la reanimación en los niños no difieren mucho con los de los adultos, sin embargo, es indispensable tener en cuenta las diferencias en cuanto a las variables hemodinámicas, el manejo de la vía aérea y la tolerancia a las pérdidas sanguíneas, el control de la temperatura y el entrenamiento y sensibilización en el manejo del paciente pediátrico.

En resumen, los cambios en la función cardiovascular en el trauma raquimedular, tanto en la fase aguda como crónica, están asociados a la respuesta adrenérgica del SNA. El conocimiento de la fisiopatología del TRM y una adecuada y objetiva valoración de la función autonómica residual (figura 3) permiten al anestesiólogo identificar de manera temprana los estados que amenazan la vida de estos pacientes y estructurar un plan de manejo oportuno. El resultado final en los pacientes con TRM es mejor cuando son manejados desde el comienzo en centros especializados, no solo por la disponibilidad del recurso tecnológico, sino por el entrenamiento del equipo de atención.

\section{LECTURAS RECOMENDADAS}

1. Casha S, Christie S. A systematic review of intensive cardiopulmonary management after spinal cord injury. J Neurotrauma 2011; 28: 1479-1495

2. Chung WS, Lin CL, Chang SN, Chung HA, Sung FC, Kao $\mathrm{CH}$. Increased Risk of Deep Vein Thrombosis and Pulmonary Thromboembolism in Patients with Spinal Cordlnjury: A Nationwide Cohort Prospective Study. Thromb Res 2014 Jan 11

3. Comerota AJ, Chouhan V, Harada RN, Sun L, Hosking J, Veermansunemi $R$, et al. The fibrinolytic effects of intermittent pneumatic compression: mechanism of enhanced fibrinolysis. Ann Surg 1997; 226: 306-13; discussion 313-314

4. Delatorre GD, Pérez J. Evaluación de Pacientes con Traumatismo Raquimedular Clínico y radiológico. Trauma 2001: 4: 22-28

5. Fehlings MG, Cadotte DW, Fehlings LN. A series of systematic reviews on thetreatment of acute spinal cord injury: a foundation for best medical practice. J Neurotrauma 2011; 28: 1329-1333

6. Furlan JC, Fehlings MG. Cardiovascular complications after acute spinal cord injury: pathophysiology, diagnosis, and management. Neurosurg Focus 2008; 25 (5)

7. García V. Trauma raquimedular. Revista de Medicina Interna y Crítica MEDICRIT 2007; 4: 66-75

8. Gunduz H, Binak DF. Autonomic dysreflexia: an important cardiovascular complication in spinal cord injury patients. Cardiol J 2012; 19: 215-219

9. Hagen EM, Rekand T, Grønning M, Færestrand S. Cardiovascular complications of spinal cord injury. Tidsskr Nor Laegeforen 2012; 132: 1115-1120
10.http://neurocirugia.univalle.edu.co/Academia/gm trauma raquimedular.htm

11. International Spinal Cord Injury Society en: http://www.iscos.org.uk/

12. Jain A, Ghai B, Jain K, Makkar JK, Mangal K, Sampley S. Severe autonomicdysreflexia induced cardiac arrest under isoflurane anesthesia in a patient with lower thoracic spine injury. J Anaesthesiol Clin Pharmacol 2013; 29: $241-243$

13. Kissoon N, Dreyer J, Walia M. Pediatric trauma: differences in pathophysiology, injury patterns and treatment compared with adult trauma. CMAJ 1990; 142: 27-34

14. Krassioukov A, Biering-Sørensen F, Donovan W, Kennelly M, Kirshblum S, Krogh K, et al. Autonomic Standards Committee of the American Spinal Injury Association/International Spinal Cord Society. International standards to document remaining autonomic function after spinal cord injury. Spinal Cord Med 2012; 35: 201-210

15. Krassioukov AV, Karlsson AK, Wecht JM, Wuermser LA, Mathias CJ, Marino RJ. Joint Committee of American Spinal Injury Association and International SpinalCord Society. Assessment of autonomic dysfunction following spinal cord injury:rationale for additions to International Standards for Neurological Assessment. J Rehabil Res Dev 2007; 44: 103-112

16. Mallek JT, Inaba K, Branco BC, Ives C, Lam L, Talving P, et al. The incidence of neurogenic shock after spinal cord injury in patients admitted to a high-volume level I trauma center. Am Surg 2012; 78: 623-626

17. Maurin $O$, de Régloix $S$, Caballé $D$, Arvis AM, Perrochon JC, Tourtier JP. Traumatic neurogenic shock. Ann Fr Anesth Reanim 2013; 32: 361-363

18. Meléndez $V$, Javiera del P. Tratamiento terapéutico kinésico a través de técnicas reeducativas vasculares en pacientes que padecen trombosis venosa profunda. http://www. efisioterapia.net/articulos/tratamientoterapeutico-kinesico-traves-tecnicas-reeducativasvasculares-pacientes-que-pad. FECHA:09 MAY 2011

19. Milligan J, Lee J, McMillan C, Klassen H. Autonomic dysreflexia: recognizing acommon serious condition in patients with spinal cord injury. Can Fam Physician 2012; 58: 831-835

20. Navarro X. Fisiología del sistema nervioso autónomo. Rev Neurol 2002; 35: 553-562

21. Popa C, Popa F, Grigorean VT, Onose G, Sandu AM, Popescu M, et al. Vascular dysfunctions following spinal cord injury. J Med Life 2010; 3: 275-285

22. Roberts A, Young WF. Prophylactic retrievable inferior vena cava filters inspinal cord injured patients. Surg Neurol Int 2010; 1:68

23. Schneck MJ. Venous thromboembolism in neurologic disease. Handb Clin Neurol 2014; 119: 289-304

24. Shen P, Luo RB, Cai SY, Zhang M. Electroversion in treatment of arrhythmia in a patient with Wolff-ParkinsonWhite syndrome and cervical spinal cord injury. Chin J Traumatol 2013; 16: 176-177

25. Sumiya T. Hypertensive intracerebral hemorrhage due to autonomic dysreflexiain a young man with cervical cord injury. J UOEH 2013; 35: 159-164

26. Top Spinal Cord InjRehabil. 2012 Summer;18(3):282-96. doi: $10.1310 /$ sci1803-282. International Standards to document remaining Autonomic Function after Spinal. Cord Injury (ISAFSCI), First Edition 2012. Contributors:, Krassioukov A, Biering-Sorensen CF, Donovan W, Kennelly M, Kirshblum S, Krogh K, Alexander MS, Vogel L, AndWecht $J$ 


\section{Autonomic Standars Assessment Form}

\begin{tabular}{|c|c|c|c|}
\hline System/Organ & Findings & Abnormal conditions & $\begin{array}{l}\text { Check } \\
\text { mark }\end{array}$ \\
\hline \multirow{6}{*}{$\begin{array}{l}\text { Autonomic } \\
\text { control of the } \\
\text { heart }\end{array}$} & Normal & & \\
\hline & \multirow[t]{3}{*}{ Abnormal } & Bradycardia & \\
\hline & & Tachycardia & \\
\hline & & Other dystrythmias & \\
\hline & Unknown & & \\
\hline & $\begin{array}{l}\text { Unable to } \\
\text { assess }\end{array}$ & & \\
\hline \multirow{6}{*}{$\begin{array}{l}\text { Autonomic } \\
\text { control of } \\
\text { blood } \\
\text { pressore }\end{array}$} & Normal & & \\
\hline & \multirow[t]{3}{*}{ Abnormal } & $\begin{array}{l}\text { Resting systolic blood pressure } \\
\text { below } 90 \mathrm{~mm} / \mathrm{hg}\end{array}$ & \\
\hline & & Orthostatic hypotensica & \\
\hline & & Autonomic dysreflexia & \\
\hline & Unknown & & \\
\hline & $\begin{array}{l}\text { Unable to } \\
\text { assess }\end{array}$ & & \\
\hline \multirow{6}{*}{$\begin{array}{l}\text { Autonomic } \\
\text { control of } \\
\text { sweating }\end{array}$} & Normal & & \\
\hline & \multirow[t]{3}{*}{ Abnormal } & Hyperhydrosis above lesion & \\
\hline & & Hyperhydrosis below lesion & \\
\hline & & Hypohydrosis below lesion & \\
\hline & Unknown & & \\
\hline & $\begin{array}{l}\text { Unable to } \\
\text { assess }\end{array}$ & & \\
\hline \multirow{5}{*}{$\begin{array}{l}\text { Temperature } \\
\text { regulations }\end{array}$} & Normal & & \\
\hline & \multirow[t]{2}{*}{ Abnormal } & Hyperthermia & \\
\hline & & Hypothermia & \\
\hline & Unknown & & \\
\hline & $\begin{array}{l}\text { Unable to } \\
\text { assess }\end{array}$ & & \\
\hline \multirow{6}{*}{$\begin{array}{l}\text { Autonomic and } \\
\text { Somatic Control } \\
\text { of Broncha- } \\
\text { pulmonary } \\
\text { System }\end{array}$} & Normal & & \\
\hline & \multirow[t]{3}{*}{ Abnormal } & $\begin{array}{l}\text { Unable to voluntarily breathe } \\
\text { requiring full ventilatory support }\end{array}$ & \\
\hline & & $\begin{array}{l}\text { Impalred voluntary breathing } \\
\text { requiring partial vent support }\end{array}$ & \\
\hline & & $\begin{array}{l}\text { Voluntary respitartion impaired } \\
\text { does not require vent support }\end{array}$ & \\
\hline & Unknown & & \\
\hline & $\begin{array}{l}\text { Unable to } \\
\text { assess }\end{array}$ & & \\
\hline
\end{tabular}

Autonomic Diagnosis: (Supraconal $\square$, Conal $\square$, Cauda Equina $\square$ )

\begin{tabular}{|c|c|c|}
\hline System/Organ & & Score \\
\hline \multicolumn{3}{|l|}{ Lower Urinary Tract } \\
\hline \multicolumn{2}{|l|}{ Arwareness of the need to emply the bladder } & \\
\hline \multicolumn{2}{|l|}{ Ability to prevent leakage (continence) } & \\
\hline \multicolumn{2}{|l|}{ Bladder emplying method (specify) } & \\
\hline \multicolumn{3}{|l|}{ Borwel } \\
\hline \multicolumn{2}{|l|}{ Sensation of need for a borwel movement } & \\
\hline \multicolumn{2}{|l|}{ Ability to present stool leakage (continence) } & \\
\hline \multicolumn{2}{|l|}{ Voluntary Sphincter contraction } & \\
\hline \multicolumn{3}{|l|}{ Sexual Function } \\
\hline \multirow[t]{2}{*}{ Genial arpusal (erection or lubrication) } & Psychogenic & \\
\hline & Reflex & \\
\hline \multicolumn{2}{|l|}{ Orgasm } & \\
\hline \multicolumn{2}{|l|}{ Ejaculation (male coly) } & \\
\hline Sensation of Menses (female only) & & \\
\hline
\end{tabular}

$2=$ Normal Function, $1=$ Reduced or Altered Neurological funtion

$0=$ Complete Loss of Control, NT $=$ Unable to assess due to preexisting or concomitant problems

Date of injury: Date of Assessment:

This form mat be freely copied and reproduced but no modified. this assesment should use the terminology found un the international SCI Data Sets (ASIA and ISCOS- http://wwwiscos.org.uk)

Examiner:

Figura 3. Evaluación de la función autonómica residual. Modificado de international Standards to document remaining Autonomic Function after Spinal Cord Injury (ISAFSCI), First Edition 2012

27. Tuli S, Tuli J, Coleman WP, Geisler FH, Krassioukov A. Hemodynamic parameters and timing of surgical decompression in acute cervical spinal cord injury. J Spinal Cord Med 2007; 30: 482-490
28. Yoo KY, Jeong CW, Kim WM, Lee HK, Kim SJ, Jeong ST, et al. Fatal cerebral hemorrhage associated with autonomic 